\title{
Isotalatizidine, a $\mathrm{C}_{19}$-diterpenoid alkaloid, attenuates chronic neuropathic pain through stimulating ERK/CREB signaling pathway-mediated microglial dynorphin A expression
}

\author{
Shuai Shao, Huan Xia, Min Hu, Chengjuan Chen, Junmin Fu, Gaona Shi, Qinglan Guo, Yu Zhou, Wenjie Wang, \\ Jiangong Shi* and Tiantai Zhang*
}

\begin{abstract}
Background: Isotalatizidine is a representative $C_{19}$-diterpenoid alkaloid extracted from the lateral roots of Aconitum carmichaelii, which has been widely used to treat various diseases on account of its analgesic, anti-inflammatory, anti-rheumatic, and immunosuppressive properties. The aim of this study was to evaluate the analgesic effect of isotalatizidine and its underlying mechanisms against neuropathic pain.

Methods: A chronic constrictive injury (CCI)-induced model of neuropathic pain was established in mice, and the limb withdrawal was evaluated by the Von Frey filament test following isotalatizidine or placebo administration. The signaling pathways in primary or immortalized microglia cells treated with isotalatizidine were analyzed by Western blotting and immunofluorescence.

Results: Intrathecal injection of isotalatizidine attenuated the CCl-induced mechanical allodynia in a dosedependent manner. At the molecular level, isotalatizidine selectively increased the phosphorylation of p38 and ERK1/2, in addition to activating the transcription factor CREB and increasing dynorphin A production in cultured primary microglia. However, the downstream effects of isotalatizidine were abrogated by the selective ERK1/2 inhibitor U0126-EtOH or CREB inhibitor of KG-501, but not by the p38 inhibitor SB203580. The results also were confirmed in in vivo experiments.
\end{abstract}

Conclusion: Taken together, isotalatizidine specifically activates the ERK1/2 pathway and subsequently CREB, which triggers dynorphin A release in the microglia, eventually leading to its anti-nociceptive action.

Keywords: Isotalatizidine, Neuropathic pain, Microglia, ERK1/2 MAPK, CREB, Dynorphin A

\section{Background}

Neuropathic pain is caused by an injury or disease in the somatosensory nervous system, including the central nerves, spinal cord, posterior root of the spinal cord, and peripheral nerves. It is highly prevalent and has an immense impact on the quality of life of patients [1]. Opiate agents and non-steroidal anti-inflammatory drugs

\footnotetext{
*Correspondence: shijg@imm.ac.cn; ttzhang@imm.ac.cn

State Key Laboratory of Bioactive Substances and Functions of Natural

Medicines, Institute of Materia Medica, Chinese Academy of Medical Sciences \& Peking Union Medical College, Beijing, China
}

are classically used to control pain, but are associated with gastrointestinal disturbances and possible risk of dependence [2]. Recently, the focus has greatly shifted to natural plant-derived antinociceptive agents with high efficacy and safety. The main and lateral roots of Aconitum carmichaelii Debx (Ranunculaceae), known as "Wutou" and "Fuzi", respectively, in Chinese, are widely used in traditional Chinese medicine as analgesic, anti-inflammatory, anti-rheumatic, and immunosuppressive agents [3-5]. More than a hundred chemicals have been isolated from the different parts of this herb [6], of which the diterpenoid

(C) The Author(s). 2020 Open Access This article is distributed under the terms of the Creative Commons Attribution 4.0 International License (http://creativecommons.org/licenses/by/4.0/), which permits unrestricted use, distribution, and 
alkaloids are the main pharmacologically active constituents. Various $A$. carmichaelii compounds were screened in an acetic acid-induced murine pain model, and the $\mathrm{C}_{19}$-diterpenoid alkaloid isotalatizidine (Fig. 1a) displayed a significant analgesic effect. However, no scientific reports have been published so far on the mechanisms underlying its analgesic properties.

Increasing evidence shows that the progression of neuropathic pain is closely related to microglial cells in the spinal cord $[7,8]$. Microglia account for only $5-12 \%$ of the cells in the central nervous system (CNS) but play a crucial role in sensing internal stimuli, transmitting excitatory signals and regulating physiological functions [9]. In addition, the microglial cells are also the resident macrophages of the CNS tissues and therefore form part of the local innate immune response [10]. Following harmful stimuli or nerve injuries, various immune cells are rapidly mobilized and activated and release chemokines and cytokines that induce peripheral sensitization and microglial activation in the peripheral and central nervous system [11, 12]. However, persistent activation of the microglial cells can elevate neuronal excitability and maintain the transmission of pain signals to the spinal dorsal horn neurons $[12,13]$. Indeed, the basis of neuropathic pain is the production of proinflammatory and pro-nociceptive mediators such as interleukins (IL-1 $\beta$, IL-6, IL-12, IL-15, IL-18) [14], IFN- $\gamma$, TNF- $\alpha$ [15], and chemokines (CCL2, CCL3, CCL4, CCL5, CCL7) $[16,17]$ by the constitutively active microglia cells $[12,18$, 19]. The mitogen-activated protein kinase (MAPKs) family of proteins, including extracellular signal-regulated kinase (ERK), p38, and c-Jun N-terminal kinase (JNK), plays a crucial role in the signaling pathways mediating microglial activation and nociceptive responses, which eventually lead to neuropathic pain $[20,21]$. Therefore, targeting the microglial signaling pathways can help understand the complex mechanisms underlying neuropathic pain and provide novel

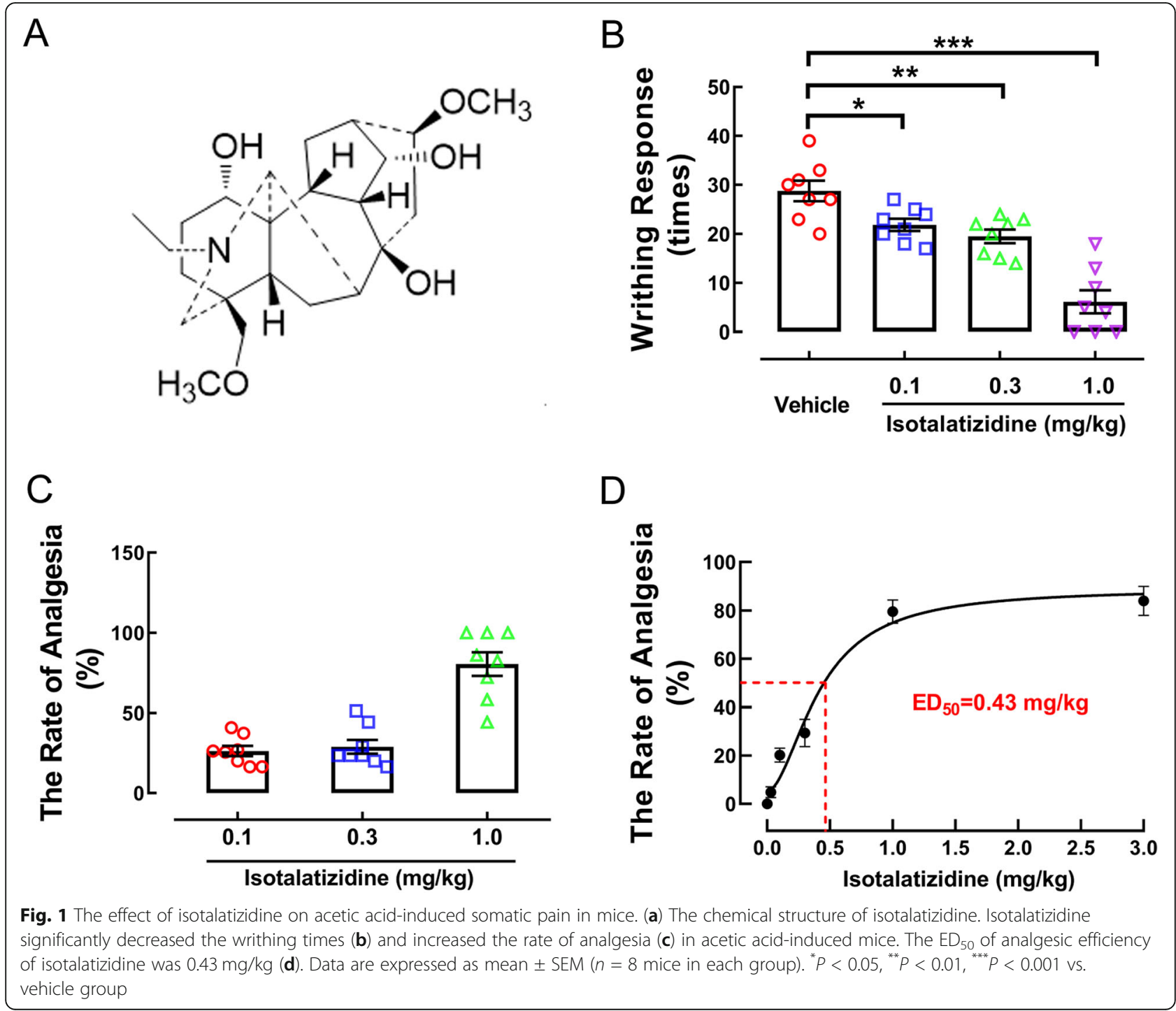


insights into drug discovery. Dynorphin A, an endogenous neurotransmitter expressed by neurons, microglia, and astrocytes, mediates neuropathic pain via its distinct $\mathrm{k}$-opioid receptor [22-24]. The production of dynorphin A involves multiple transcription factors [25, 26], including the cAMP response element-binding protein (CREB) which induces the transcription of the dynorphin A precursor prodynorphin [27]. As an upstream regulator of CREB, phosphorylated MAPK is crucial to prodynorphin expression and dynorphin A release in microglia. Although dynorphin A is elevated during neuropathic pain, it is not clear whether it is pro- or anti-nociceptive.

The aim of the present study was to evaluate the antinociceptive effects of isotalatizidine and explore the relevant signaling pathways in microglial cells, in order to determine its possible mechanism against neuropathic pain.

\section{Materials and methods}

\section{Drugs and reagents}

Isotalatizidine was extracted and purified by the Institute of Materia Medica, Chinese Academy of Medical Sciences and Peking Union Medical College, and the purity was validated as > 95\% using high-performance liquid chromatography. For the experiments, it was dissolved in dimethyl sulfoxide (DMSO) and subsequently diluted in sterile saline (0.9\%). SB203580 and U0126-EtOH were all purchased from TargetMol (Shanghai, China). KG501 was purchased from MedChemExpress (Shanghai, China) and dissolved in DMSO, and diluted with DMEM, DMEM/F-12, or saline before use. The cell culture reagents were purchased from the Invitrogen Corporation (Thermo Fisher Scientific, Carlsbad, CA, USA). Anti-dynorphin A antibody was purchased from Abcam (Cambridge, UK), and the Alexa 546-conjugated goat anti-rabbit and Alexa 488-conjugated goat anti-mouse secondary antibodies from the Life Technology (Thermo Fisher Scientific, Carlsbad, CA, USA). The remaining antibodies were purchased from the Cell Signaling Technology (Beverly, MA, USA). The goat serum was purchased from the Beyotime Biotechnology (Shanghai, China), and triton X-100 from the Sigma Aldrich.

\section{Experimental animals}

The ICR mice (female, weighing18-20 g) and C57BL/6 mice (females, $18-20 \mathrm{~g}$ ) were obtained from the Beijing Huafukang Experimental Animal Institute (Beijing, China). The adult mice (5-6 per cage) were housed at room temperature $\left(22 \pm 2{ }^{\circ} \mathrm{C}\right)$ in specific pathogen-free conditions under a 12/12-h reversed light-dark cycle, with food and water provided ad libitum. The mice were acclimatized for 3-4 days before the experiments and randomly divided into the different groups. Animal studies were conducted following the protocols approved by the Experimental Animal Welfare and Ethics Committee of the Institute of
Materia Medica, Chinese Academy of Medical Sciences. Animal studies are reported in compliance with the ARRIVE guidelines [28]. The experimental designs were based on the rule of the replacement, refinement, and reduction to reduce suffering of the animals and use the minimum number of animals.

\section{Establishment of somatic or neuropathic pain model and treatment \\ Acetic acid-induced abdominal writhing test}

Acetic acid-induced mouse somatic pain model was used to evaluate the analgesic effect of isotalatizidine. ICR mice were pre-treated with isotalatizidine $(0.1,0.3$, or $1.0 \mathrm{mg} /$ $\mathrm{kg}$ ) or vehicle (normal saline, $1 \mathrm{ml} / \mathrm{kg}$ ) by a single intraperitoneal injection. Thirty minutes later, obtained $1.0 \%$ acetic acid solution $(10 \mathrm{ml} / \mathrm{kg})$ was injected intraperitoneally. The times of writhes and stretching were counted over a period of $15 \mathrm{~min}$ after acetic acid injection.

\section{Chronic constrictive injury (CCI)-induced neuropathic pain model test}

Chronic neuropathic pain following peripheral nerve injury was simulated by chronic constrictive injury (CCI) of the unilateral sciatic nerve as described previously [29]. Briefly, the C57BL/6 mice were anesthetized with isoflurane and randomly divided into the sham-operated, untreated CCI model, and isotalatizidine-treated (0.1, 0.3, and $1 \mathrm{mg} / \mathrm{kg}$ ) groups $(n=6$ each). The left sciatic nerve trunk was exposed by blunt dissection at mid-thigh level, and 4 ligatures (4-0 chromic catgut) were tied loosely around the nerve with $1 \mathrm{~mm}$ spacing. The control mice were subjected to sham surgery wherein the sciatic nerve was only exposed but not ligated.

\section{Intrathecal injection}

On the eighth day after surgery, the mice were given a single intrathecal injection of the suitable isotalatizidine dose or saline as described previously by Hylden and Wilcox [30] with slight modifications. Briefly, the mice were anesthetized with isoflurane ( $4 \%$ for induction and $1 \%$ for maintenance), and a $100 \mu \mathrm{l}$ micro-injector was inserted from the intervertebral space between the L5 and L6 spinal cord into the spinal subarachnoid space. After confirming proper intrathecal injection by tail flicking, $100 \mu \mathrm{l}$ normal saline or drug was microinjected followed by a $100-\mu \mathrm{l}$ normal saline flush.

\section{Behavioral analysis}

Acetic acid-induced writhing was evaluated by counting the number of writhes and stretches over $15 \mathrm{~min}$ after its injection. The sensitivity of mechanical nociception in the CCI model was measured in terms of the withdrawal threshold of the ipsilateral and contralateral limbs by the Von Frey test (Von Frey filaments, IITC 
Life Science Inc, California, USA) after $30 \mathrm{~min}, 1 \mathrm{~h}, 2 \mathrm{~h}$, and $4 \mathrm{~h}$ of intrathecal injection. The animals were acclimatized in boxes set on an elevated metal mesh floor for at least $30 \mathrm{~min}$. A series of monofilaments of different pressure values were pressed vertically on the sole of the hind paws with an increasing force till the animal withdrew the hindlimb. The procedure was repeated 5 times, and the average threshold value was calculated. All behavioral analyses were performed by an investigator blinded to the experimental grouping.

\section{BV-2 and primary microglial cell culture}

BV-2 cells were cultured in Dulbecco's modified Eagle's medium (DMEM) supplemented with 10\% fetal bovine serum (FBS), $100 \mathrm{U} / \mathrm{ml}$ penicillin, $100 \mu \mathrm{g} / \mathrm{m}$ streptomycin, and $5.5 \mathrm{mM}$ glucose at $37{ }^{\circ} \mathrm{C}$ under $5 \% \mathrm{CO}_{2}$ and $95 \% \mathrm{hu}-$ midity. To isolate primary microglia cells, spinal cords were removed from 1-day-old Wistar rats (Beijing Huafukang Experimental Animal Institute) and minced in icecold D-Hank's medium containing penicillin $(100 \mathrm{U} / \mathrm{ml})$ and streptomycin $(100 \mu \mathrm{g} / \mathrm{ml})$. After digesting with $0.125 \%$ trypsin, the dissociated cells were suspended in equal volume of complete DMEM/F12 medium (supplemented with $10 \% \mathrm{FBS}, 100 \mathrm{U} / \mathrm{ml}$ penicillin and $100 \mu \mathrm{g} / \mathrm{ml}$ streptomycin) to stop the reaction. The cell suspension was then filtered through a $200-\mu \mathrm{m}$ mesh screen to remove tissue debris and centrifuged at $1000 \mathrm{rpm}$ for $5 \mathrm{~min}$. The pellet was re-suspended in DMEM/F12 medium and plated onto poly-L-lysine pre-coated $(100 \mu \mathrm{g} / \mathrm{ml}) 75-\mathrm{cm}^{2}$ tissue culture flasks. The primary microglial cells were cultured at $37{ }^{\circ} \mathrm{C}$ under $5 \% \mathrm{CO}_{2}$ for 10 days and harvested by shaking the flasks at $180 \mathrm{rpm}$ for $5 \mathrm{~h}$. Multiple aliquots were then replated, and unattached cells were removed by washing with serum-free DMEM. The final harvested microglial cells were identified by IBA-1 immunoreactivity and exhibited > 95\% purity.

\section{RNA isolation and qRT-PCR}

Total RNA was extracted from spinal dorsal lumbar enlargements and BV-2 in TRIzol (Invitrogen, Carlsbad, CA, USA) on ice and then reverse transcribed into cDNA and subjected to qPCR. QPCR was performed with Roche LightCycler 480 PCR Detection System (Roche, Switzerland). The fold change was calculated using the $2^{-\triangle \Delta C t}$ method after normalization to GAPDH. The primer sequences are as follows: GAPDH (5'-ATC CCA TCA CCA TCT TCC AGG AG-3' and 5' CCT GCT TCA CCA CCT TCT TGA TG $3^{\prime}$ ) and Prodynorphin (5' ${ }^{\prime}$-CGG AAC TCC TCT TGG GGT AT-3' and 5'-CGG AAC TCC TCT TGG GGT AT-3').

\section{Protein extraction and Western blotting}

BV-2 and primary microglia cells were seeded into 12well plates at the density of $2 \times 10^{6}$ cells per well. After overnight culture, the cells were pre-treated with $25 \mu \mathrm{M}$ isotalatizidine for $1 \mathrm{~h}$, harvested, and lysed to extract protein. The spinal dorsal lumbar enlargements were separated from CCI mice after measurement of mechanical withdrawal threshold and then lysed to extract protein. The protein concentration in the cell and tissue lysates were determined by BCA Protein Assay Kit (Thermo Fisher Scientific, Carlsbad, CA, USA), and $20 \mu \mathrm{g}$ of proteins per sample was separated by $8 \%$ sodium dodecyl sulfate-polyacrylamide gel electrophoresis (SDS-PAGE). The bands were transferred to a polyvinylidene fluoride (PVDF) membrane (Millipore Corp., Bedford, MA, USA) that was then blocked with $5 \%$ bovine serum albumin (BSA) in tris-based saline-Tween 20 (TBST) at room temperature for $1 \mathrm{~h}$. After incubating overnight with the primary antibodies against p-p38, pERK, p-JNK, p-CREB, p38, ERK, JNK, CREB, dynorphin A (all diluted 1:1000), $\beta$-tubulin, and GAPDH (1:5000 each) at $4{ }^{\circ} \mathrm{C}$, the blots were then incubated with horseradish peroxidase-conjugated goat anti-rabbit and goat anti-mouse secondary antibodies [31]. The membrane was developed using enhanced chemiluminescence reagents (Perkinelmer, USA), and the bands were visualized with Tanon 2000 Imaging system (Beijing, China) and their intensities were quantified using ImageJ Software (NIH, USA).

\section{Dynorphin A detection}

The spinal cord was washed with ice-cold saline and the spinal dorsal lumbar enlargements rapidly dissected and then immediately frozen in liquid nitrogen. Thawed tissue with ice-cold saline was disrupted using a protein homogenizer and centrifugated at $12,000 \times g$ for $15 \mathrm{~min}$ at $4{ }^{\circ} \mathrm{C}$. Protein concentrations were determined by the use of the BCA Protein Assay Kit (Thermo Fisher Scientific, Carlsbad, CA, USA) with bovine serum albumin as a standard. Dynorphin A immunoassay was performed using a commercial enzyme-linked immunosorbent assay (ELISA) kit (Jiangsu Meimian Co., Nanjing, China) according to the instruction. Standard curves were constructed using known concentrations of dynorphin A by a non-linear regression analysis (Prism, GraphPad Inc, San Diego, CA). The dynorphin A content in the spinal cord extracts was determined from the standard curve done in parallel assays.

\section{Immunofluorescence}

Primary microglia were seeded into a 12-well plate $(2 \times$ $10^{6}$ cells per well $)$ pre-coated with poly-L-lysine $(100 \mu \mathrm{g} /$ $\mathrm{ml}$ ) and cultured overnight. After treating with isotalatizidine or MAPK inhibitors, the cells were fixed with $4 \%$ paraformaldehyde for $30 \mathrm{~min}$ at room temperature and permeabilized with $0.2 \%$ triton $\mathrm{X}-100$ in phosphatebuffered saline (PBS) containing 10\% goat serum for $1 \mathrm{~h}$. 
The cells were then incubated overnight with antidynorphin A (1:200) and anti-IBA-1 (1:200) primary antibodies at $4{ }^{\circ} \mathrm{C}$, washed with PBS, and then incubated with goat anti-rabbit Alexa Fluor 546- and goat antimouse Alexa Fluor 488-conjugated secondary antibody (1:200) for $1 \mathrm{~h}$ at $37^{\circ} \mathrm{C}$. The nuclei were counterstained with 4, 6-diamidino-2-phenylindole $(1 \mu \mathrm{g} / \mathrm{ml}$, SigmaAldrich), washed thrice with PBS, and imaged using Cytation 5 imaging reader (BioTek, VT, USA) [32].

\section{Statistical analysis}

Data were presented as the mean $\pm \mathrm{SEM}$, and $p<0.05$ was considered statistically significant. The $50 \%$ mechanical withdrawal threshold (MWT (g)) in the Von Frey test was calculated as $10^{[\log (f \cdot 10000)+k \delta]} / 10000$ and compared by two-factor analysis of variance (ANOVA) followed by Tukey's post hoc test using Prism (version 5.01, GraphPad Software Inc., San Diego, CA, USA). The other experiments were analyzed using one-way ANOVA followed by an appropriate post hoc test. The in vitro assays were performed at least thrice.

\section{Results}

Isotalatizidine attenuates pain hypersensitivity of somatic pain

The analgesic effect of isotalatizidine was tested by the acetic acid-induced writhing test, which showed that this drug significantly reduced the number of writhes compared to the placebo in a dose-dependent manner (Fig. 1b). The analgesic efficiencies were $26.37 \%, 30.43 \%$, and $76.23 \%$ at the doses of $0.1,0.3$, and $1 \mathrm{mg} / \mathrm{kg}$, respectively, (Fig. 1c). Accordingly, the analgesic $\mathrm{ED}_{50}$ of isotalatizidine was calculated to be $0.43 \mathrm{mg} / \mathrm{kg}$ (Fig. 1d).
Isotalatizidine treatment alleviated CCl-induced neuropathic pain

We next determined its effects on mechanical allodynia in a CCI-induced neuropathic pain model by evaluating limb withdrawal. As shown in Fig. 2a, the mechanical withdrawal threshold of the ipsilateral but not the contralateral paw decreased gradually after sciatic nerve ligation and reached a lower level on the eighth day post-surgery. However, isotalatizidine increased this threshold in the ipsilateral paw in a dose-dependent manner compared to the placebo-treated mice, but had no effect on the contralateral paw (Fig. 2b). In addition, no apparent sedative or motor side effects of isotalatizidine were observed during the entire treatment duration.

Isotalatizidine promoting the phosphorylation of p38 and ERK $1 / 2$ in microglial cells but not JNK

MAPK family includes three different signaling cascades of p38, ERK1/2, and JNK, and the phosphorylation of MAPKs plays a critical role in the activation of microglial cells. To investigate the effects of isotalatizidine on MAPK signal activation and selectivity for members of MAPKs, primary microglial and BV-2 cells were applied to test the phosphorylated level of p38, ERK1/2, and JNK under the selective inhibitors by western blotting. The results showed that isotalatizidine treatment at 25 $\mu \mathrm{M}$ for $1 \mathrm{~h}$ significantly increased the phosphorylation of p38 $(p<0.01$, Fig. 3a-c) and ERK1/2 $(p<0.001$, Fig. 3a, $\mathrm{b}$, and $\mathrm{d}$ ) both in cultured primary microglia and BV-2 cells. However, the same concentration and treatment of iaotalatizidine had no significant change on level of JNK phosphorylation both in cultured primary microglia and BV-2 cells (Fig. 3a, b, and e).

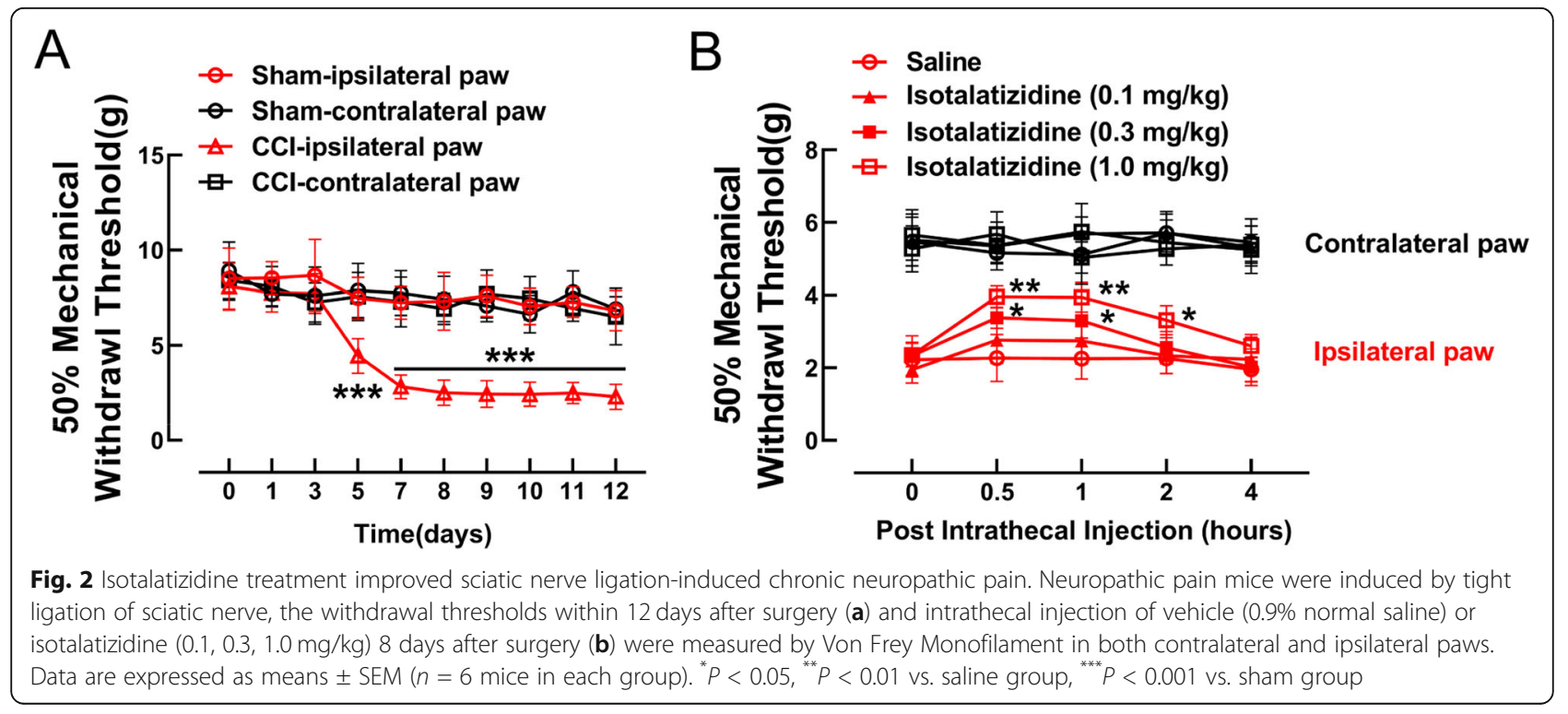




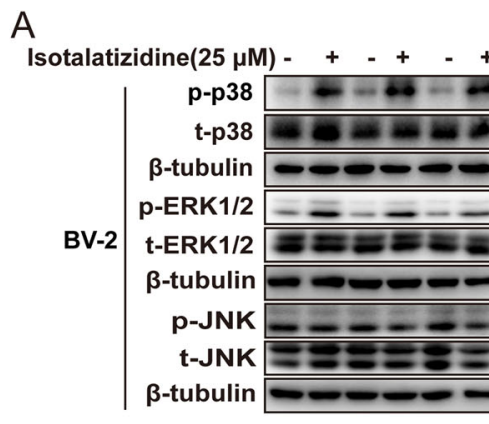

C

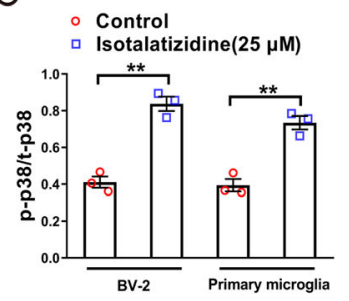

$\mathbf{F}$

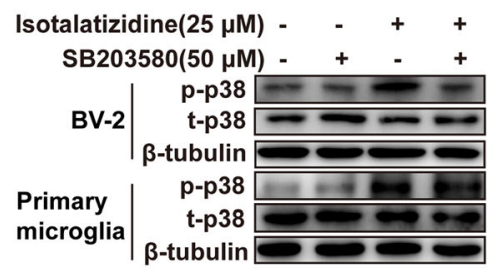

$\mathrm{H}$

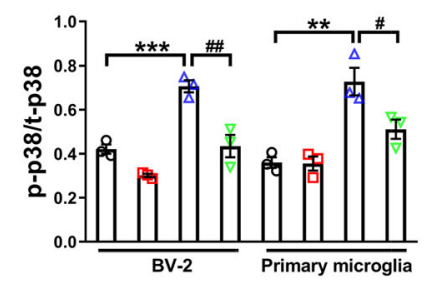

Isotalatizidine $(25 \mu \mathrm{M})-\quad++\quad-++$

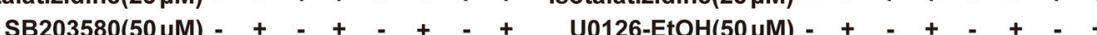

B

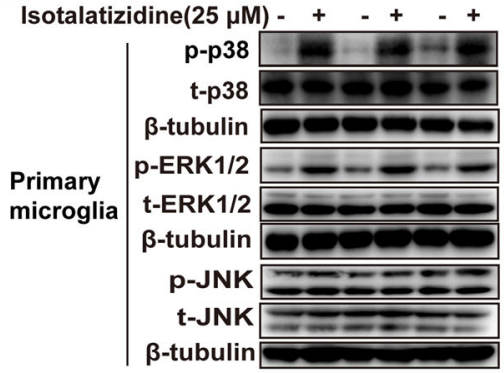

$\mathrm{E}$

Control
Isotalatizidine $(25 \mu \mathrm{M})$

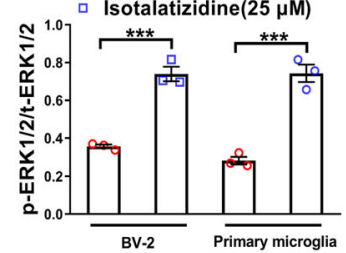

口. Isotalatizidine $(25 \mu \mathrm{M})$

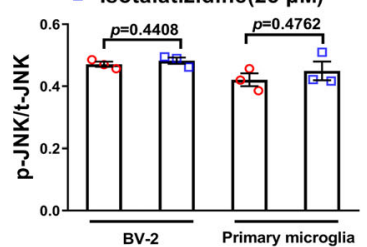

G
I
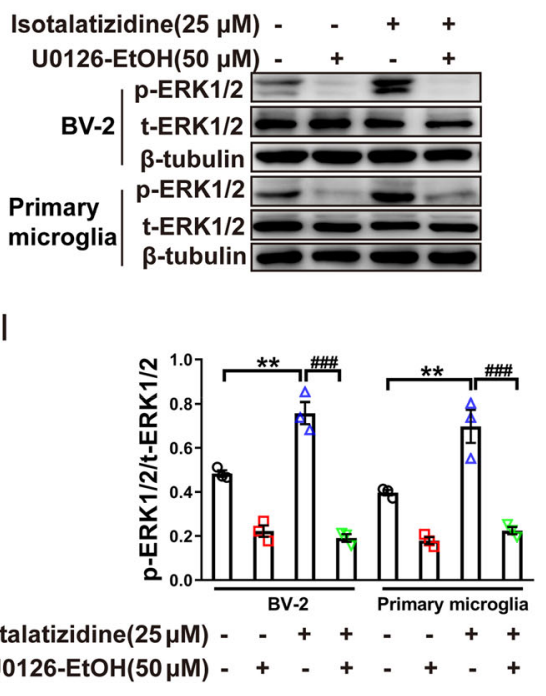

Fig. 3 Effects of isotalatizidine on phosphorylation of p38, ERK1/2, and JNK in BV-2 and primary microglial cells. Effects of isotalatizidine on phosphorylation of p38, ERK1/2, and JNK in BV-2 (a) and primary microglial cells (b). Isotalatizidine (25 MM) obviously promoted the phosphorylation of p38 (c) and ERK1/2 (d), but not JNK (e) in cultured microglial cells. Selective p38 inhibitor SB $203580(\mathbf{f}, \mathbf{h})$ and ERK1/2 inhibitor $\mathrm{U} 0126-\mathrm{EtOH}(\mathbf{g}, \mathbf{i})$ significantly blocked the isotalatizidine activated phosphorylation of p38 or ERK1/2, respectively. The data are expressed as mean \pm SEM of three independent experiments. ${ }^{* *} P<0.01,{ }^{* * *} P<0.001$ vs. control group; ${ }^{\#} P<0.05$, ${ }^{\# \#} P<0.01$, ${ }^{\# \# \#} P<0.001$ vs. isotalatizidine group

To further explore the causal relationship of MAPK members with isotalatizidine, the selective MAPK subtype inhibitors were applied to determine the responsible subtypes for isotalatizidine. Pre-treatment of the microglial cells with the p38 inhibitor SB203580 $(50 \mu \mathrm{M})$ and ERK1/ 2 inhibitor U0126-EtOH $(50 \mu \mathrm{M})$ significantly abrogated the effects of isotalatizidine (Fig. 3f-i). These results strongly suggested that the analgesic effect of isotalatizidine is likely related to the activation of p38 and the ERK1/2 MAPK signaling pathways in the microglia.
Isotalatizidine stimulating the CREB activation and dynorphin A release via ERK1/2 pathway in the primary microglial cells

Given the important role of MAPK in CREB activation, we investigated the relationship between phosphorylation of CREB and isotalatizidine in primary microglia. To confirm the correlation between CREB changes with subtype of MAPKs, the selective p38 inhibitor of SB203580 or ERK1/2 inhibitor of U0126-EtOH were used in this assay. As shown in Fig. 4, treatment with isotalatizidine at concentration of 


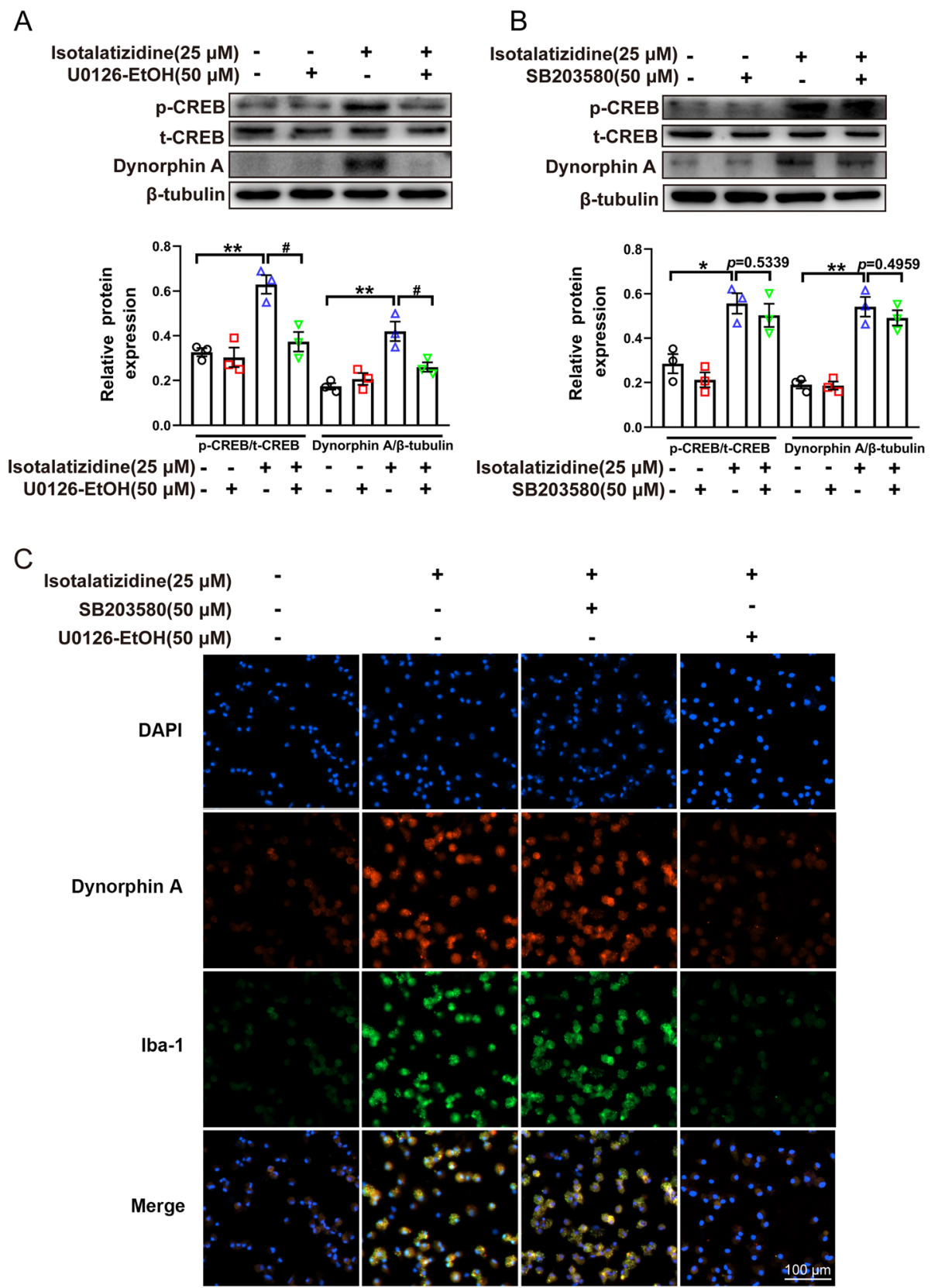

Fig. 4 Isotalatizidine stimulating the CREB activation and dynorphin A expression via ERK1/2 pathway in the primary microglial cells. In cultured primary microglial cells, isotalatizidine $(25 \mu \mathrm{M})$ markedly induced the phosphorylation of CREB and expression of dynorphin $A$, and the phosphorylated levels of CREB and increased dynorphin A level were markedly prevented by ERK1/2 inhibitor of U0126-EtOH (a) but not p38 inhibitor of $\mathrm{U} 0126-\mathrm{EtOH}(\mathbf{b})$. Immunofluorescence analysis showed that isotalatizidine-stimulated IBA-1 expression and dynorphin A production also could be blocked by ERK1/2 inhibitor of SB203580 (c). The data are expressed as mean \pm SEM $(n=3) .{ }^{*} P<0.05,{ }^{* *} P<0.01$ vs. control group; ${ }^{\#} P<0.05$ vs. isotalatizidine group

$25 \mu \mathrm{M}$ induced an obvious phosphorylation of CREB in primary microglial cells. However, the phosphorylated levels of CREB elevated by isotalatizidine were markedly prevented by ERK1/2 inhibitor of U0126-EtOH (Fig. 4a, c) but not p38 inhibitor of SB203580 (Fig. 4b, c) in cultured primary microglial cells at $50 \mu \mathrm{M}$ concentration. The results implied that isotalatizidine-increased phosphorylated level of CREB might be mediated by ERK1/2 MAPK. Next, under the same conditions, the release of dynorphin A was determined by immunofluorescence assay and Western blotting. The results indicated that only ERK1/2 inhibitor of U0126-EtOH obviously reduced the increased level of dynorphin A by isotalatizidine (Fig. 4a, c). However, p38 inhibitor of SB203580 had no effect for the isotalatizidine-induced dynorphin A 
release (Fig. 4b, c). The above results implied that isotalatizidine-stimulated phosphorylated CREB and dynorphin A release might be mediated by ERK1/2 MAPK.

Isotalatizidine exerts its analgesic effect by increasing dynorphin A production in activated spinal microglial cells via the ERK1/2-CREB pathway

To explore whether the analgesic effect of isotalatizidine in chronic neuropathic pain mice is mediated by ERK1/2CREB-dynorphin A axis, CCI mice model was established to measure the relationship between mechanical withdrawal threshold and the activation of ERK1/2-CREB pathway. As shown in Fig. 5, isotalatizidine $(1 \mathrm{mg} / \mathrm{kg})$ treatment by intrathecal injection induced a time-dependent mechanical anti-allodynia in the ipsilateral paws, which was completely prevented by the pretreatment of ERK1/2 inhibitor of U0126-EtOH or CREB inhibitor of KG-501, respectively, but not p38 inhibitor of SB203580 (Fig. 5a). Further investigating indicated that isotalatizidine induced a significant phosphorylation of ERK1/2 and CREB and release of dynorphin A in spinal dorsal tissue in CCI mice (Fig. 5b). Our results further displayed that the elevated level of phosphorERK1/2 and phosphor-CREB and expression of dynorphin A were all blocked by selective REK1/2 inhibitor of U0126EtOH. Meanwhile, selective CREB inhibitor of KG-501 also could suppress the phosphorylated level of CREB and expression of dynorphin A induced by isotalatizidine, but not the change of phosphor-ERK1/2. It is known that only

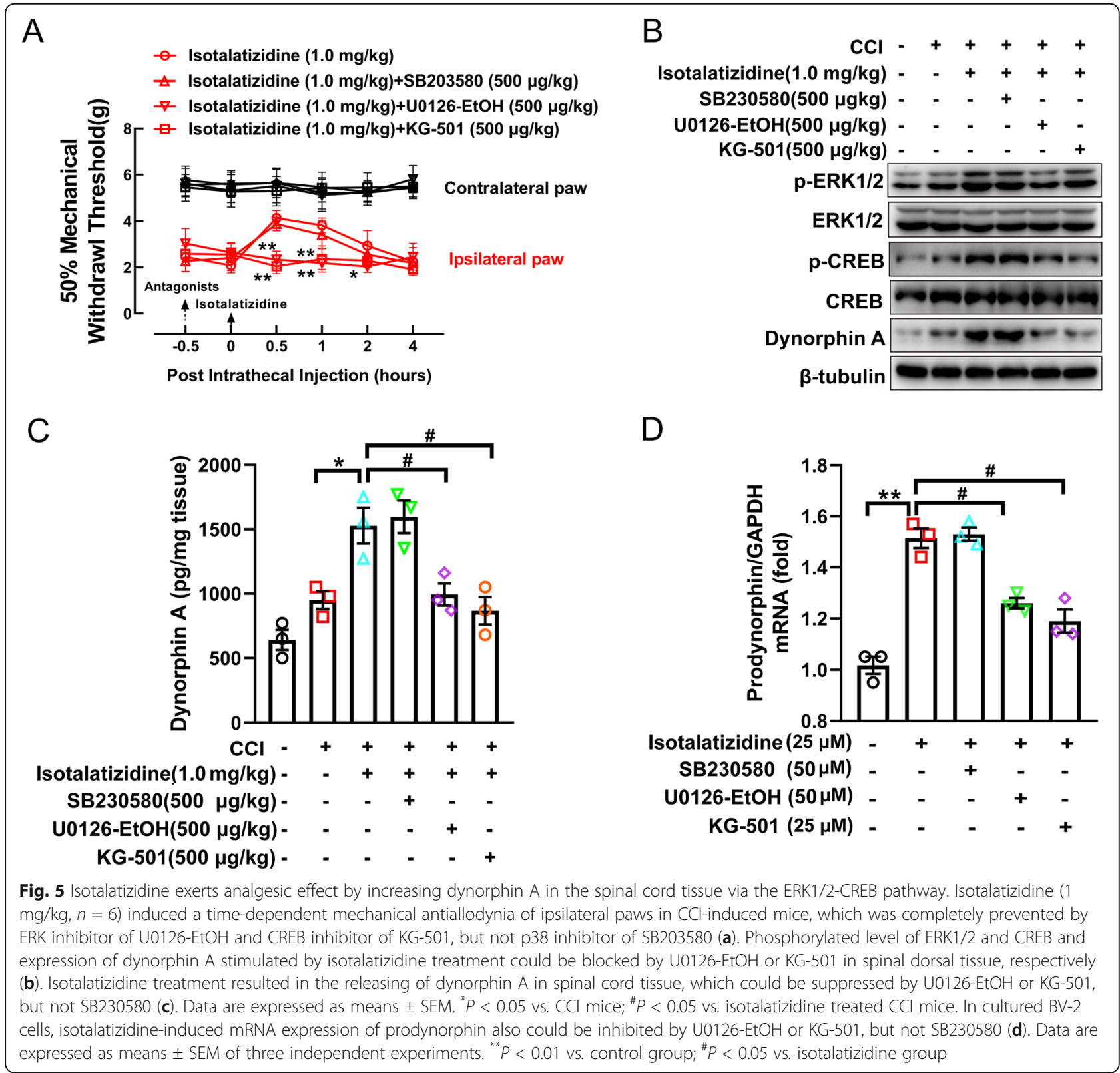


secreted dynorphin A binds to opioid receptor inducing the regulatory effect. Therefore, the level of dynorphin A was detected by ELISA in spinal dorsal tissue, the results showed that isotalatizidine significantly increased the secretion of dynorphin $\mathrm{A}$, and this elevation could be blocked by ERK1/2 inhibitor of U0126-EtOH or CREB inhibitor of KG-501, respectively (Fig. 5c). To further conform the relationship between the production of dynorphin $\mathrm{A}$ and ERK1/2-CREB signal, the mRNA expression of prodynorphin, precursor of dynorphin A, also was measured in microglia of BV-2. The data indicated that isotalatizidine increased the expression of prodynorphin in BV-2 cell, and these elevated expression of prodynorphin could be prevented by ERK1/2 inhibitor of U0126-EtOH or CREB inhibitor of KG-501, but not the p38 inhibitor of SB230580 (Fig. 5d). Taken together, our data demonstrated that isotalatizidine exerts analgesic effects by releasing dynorphin A which is mediated by activating the ERK1/2-CREB pathway in microglial cells.

\section{Discussion}

Our study shows for the first time that isotalatizidine extracted from $A$. carmichaelii can effectively block the mechanical allodynia in a CCI-induced neuropathic pain model, as well as suppressing writhing in a somatic pain model. Mechanistically, isotalatizidine might induce the phosphorylation of ERK1/2 and CREB, which results in the production of dynorphin A in microglia and exerts analgesic effect.

The lateral root of A. carmichaelii Debx has been used for centuries in China, Japan, and Korea to relieve rheumatoid arthritis pain. It has also shown pharmacological effects against cardiovascular diseases, rheumatic fever, joint pain, bronchial asthma, gastroenteritis, collapse, syncope, diarrhea, and edema [33]. Isotalatizidine is a $\mathrm{C}_{19}$-diester and monoester diterpenoid alkaloid belonging to the amine alcohol type that is extracted from the lateral roots of $A$. carmichaelii and exhibits low toxicity and potent analgesic action. However, no study so far has shown the antinociceptive effect of isotalatizidine in a neuropathic pain model. We observed that isotalatizidine not only relieved acetic acid-induced somatic pain in a mouse model but also alleviated mechanical allodynia in the ipsilateral paw of a CCI-induced neuropathic pain model in a dosedependent manner, without affecting the normal nociceptive response in the contralateral paw.

The activation of spinal microglia plays an important role in initiating and sustaining chronic neuropathic pain, with the intervention of the MAPK signaling pathway. Activated MAPKs induce diverse intracellular response and are also involved in maintaining hypersensitivity in neuropathic pain via transcriptional and non-transcriptional

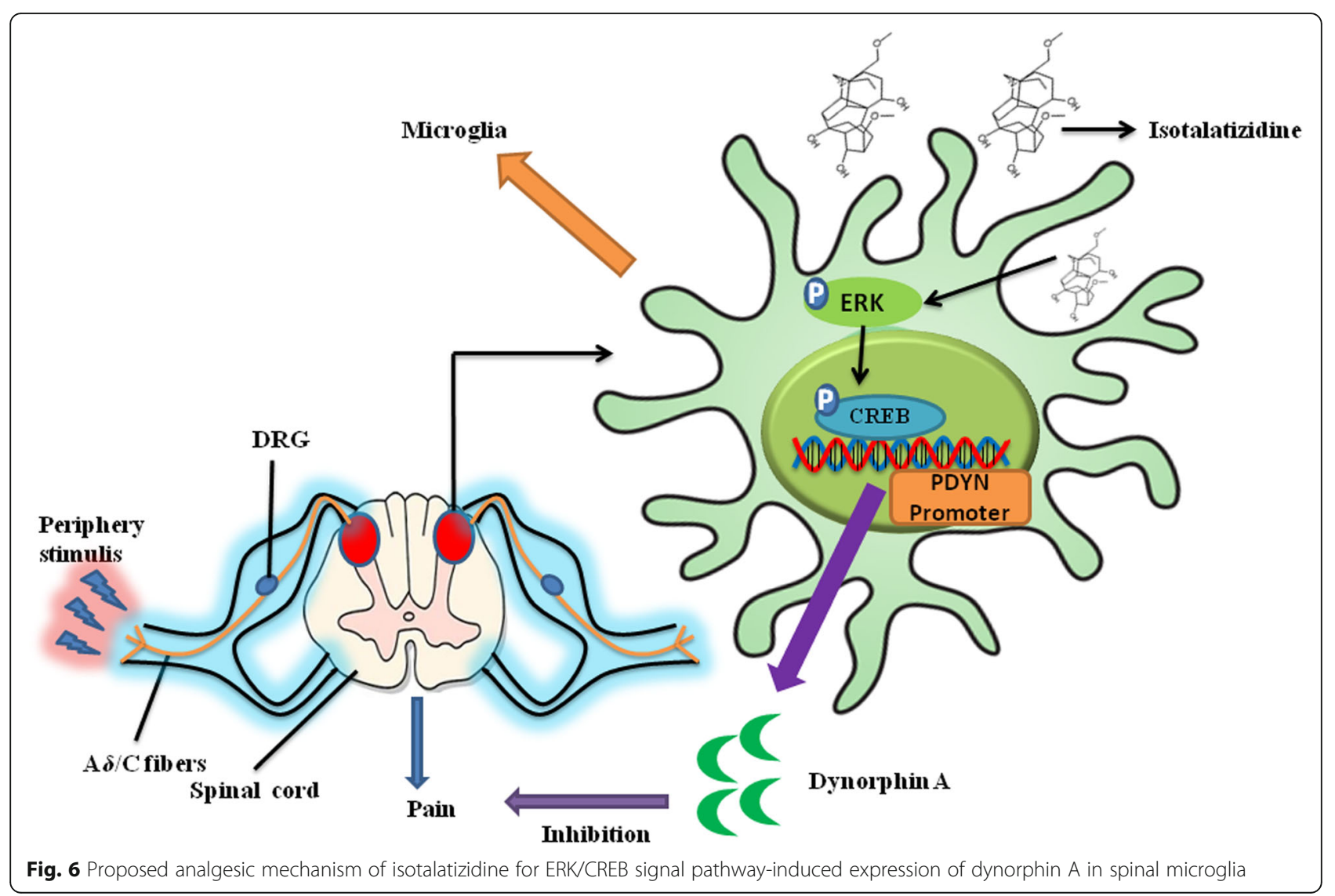


regulation of downstream factors [21, 34]. Consistent with this, MAPKs and microglial activation inhibitors effectively attenuate neuropathic pain in different models [35]. We found that isotalatizidine stimulated p38 and ERK1/2 in cultured BV-2 cell line or primary microglia, which was completely inhibited by the respective inhibitors. Our findings are consistent with that of Huang et al. who demonstrated that cynandione $\mathrm{A}, \mathrm{a}_{19}$-diester and monoester diterpenoid alkaloid structurally similar to isotalatizidine and extracted from Cynanchi Wilfordii Radix, exerted its anti-nociceptive effect by non-selectively activating MAPKs [36]. We hypothesized therefore that the activation of p38 or ERK1/2 mediated the analgesic effect of iaotalatizidine in the neuropathic pain model.

As a downstream target of MAPK, CREB plays a pivotal role in the development of neuropathic pain by regulating transcription and secretion of diverse neurotransmitters. Binding of cAMP to the regulatory subunit of protein kinase A (PKA) phosphorylates CREB, which eventually regulates multiple cellular events. Previous studies have shown that activated p38 or ERK1/2 induces CREB phosphorylation in microglial or neuronal cells [37, 38]. Consistent with this, isotalatizidine increased the levels of $\mathrm{p}$-CREB in microglial cells, which was blocked by inhibiting ERK1/2 but not p38 or JNK. This suggested that isotalatizidineinduced phosphorylation of CREB was specifically mediated by the ERK1/2 pathway.

As an endogenous ligand, dynorphin A is closely related to neuropathic pain by binding to the $\mathrm{k}$-opioid receptor, but the detailed antinociceptive mechanism of dynorphin A is still unclear [39, 40]. Elevated dynorphin A has previously been implicated in the development and maintenance of neuropathic pain, since intrathecal administration of antidynorphin A serum ameliorated pain in an animal model [41]. However, several studies showed that exogenous agents could induce the production of dynorphin A by microglia or neurons leading to an analgesic effect in CCIinduced model [42-44]. Further investigating indicated that the analgesic effect by dynorphin A failed to administrate specific anti-dynorphin A antibody or selective k-opioid receptor antagonist GNTI. In the present study, we also found that isotalatizidine induced the secretion of dynorphin A in the spinal cord tissue in CCI-induced neuropathic pain model, and this elevated dynorphin A assuages rather than aggravates neuropathic pain. Furthermore, we found that the isotalatizidine-induced secretion of dynorphin A could be blocked by the selective ERK1/2 inhibitor or selective CREB inhibitor, but not p38 inhibitor. Further experimental also supported the above results, which indicated that isotalatizidine induced a mRNA expression of prodynorphin in cultured microglia of BV-2, and this increased expression also could be prevented by ERK1/2 inhibitor or CREB inhibitor, respectively. Taken together, the anti-nociception action of isotalatizidine in CCI-induced neuropathic pain model is mediated via the activation of the ERK1/2-CREB-dynorphin A axis. The proposed signal transduction pathway is presented in Fig. 6. Our findings provide novel insights into the pathological role of microglial activation in neuropathic pain and the underlying mechanisms.

\section{Conclusion}

Isotalatizidine alleviated mechanical allodynia in neuropathic pain in a dose-dependent manner by activating the ERK1/2 pathway and phosphorylating CREB, which triggered dynorphin A release from the microglia. Our findings provided primary pharmacological evidence for the potential use of isotalatizidine against neuropathic pain.

\section{Abbreviations}

CCl: Chronic constrictive injury; CNS: Central nervous system; CREB: CAMPresponse element binding protein; ERK: Extracellular signal-regulated kinase; JNK: c-Jun N-terminal kinase; MAPK: Mitogen-activated protein kinase; PBS: Phosphate-buffered saline

\section{Acknowledgements}

Not applicable.

\section{Authors' contributions}

SS and TZ designed the research study and wrote the paper. $\mathrm{XH}, \mathrm{GQ}$, and SJ isolated the compound. SS, HM, CC, FJ, SG, and ZY performed the experiment. WW analyzed the data. All authors read and approved the final manuscript.

\section{Funding}

This work was supported by the National Natural Science Foundation of China (No. 81573445, 81973338, 81630094), Beijing Natural Science Foundation (No. 7182115), Drug Innovation Major Project of China, China (No. 2018ZX09711001-003-001), and CAMS Innovation Fund for Medical Science, China (No. 2017-I2M-3-011 and 2017-12M-3-010).

Availability of data and materials

The datasets during and/or analyzed during the current study are available from the corresponding author on reasonable request.

Ethics approval and consent to participate

Not applicable.

Consent for publication

Not applicable.

\section{Competing interests}

The authors declare that they have no competing interests.

Received: 30 September 2019 Accepted: 30 December 2019

Published online: 10 January 2020

\section{References}

1. Tan T, Barry PW, Reken S, Baker M. Pharmacological management of neuropathic pain in non-specialist settings: summary of NICE guidance. BMJ. 2010;340:707-9.

2. Melnikova I. Pain market. Nat Rev Drug Discov. 2010;9:589-90.

3. Guo QL, Xia H, Shi GN, Zhang TT, Shi JG. Aconicarmisulfonine A, a sulfonated C20-diterpenoid alkaloid inner salt with skeleton and analgesic activity from an aqueous extract of the lateral roots of Aconitum carmichaelii. Org. lett. 2018;20:816-9.

4. Guo QL, Xia H, Meng XH, Shi GN, Xu CB, Zhu CG, Zhang TT, Shi JG. C19 Diterpenoid alkaloid arabinosides from an aqueous extract of the lateral root of Aconitum carmichaelii and their analgesic activities. Acta. Pharm. Sin. B. 2018;8:409-19. 
5. Wu YZ, Shao S, Guo QL, Xu CB, Xia H, Zhang TT, Shi JG. Aconicatisulfonines $A$ and $B$, analgesic zwitterionic C20-diterpenoid alkaloids with a rearranged atisane skeleton from Aconitum carmichaelii. Org. lett. 2019;21:6850-4.

6. Zhang J, Sun GB, Lei QF, Li GZ, Wang JC, Si JY. Chemical constituents of lateral roots of Aconitum carmichaelii Debx. Yao Xue Xue Bao. 2014;49: 1150-4.

7. Taves S, Berta T, Chen G, Ji RR. Microglia and spinal cord synaptic plasticity in persistent pain. Neural Plast. 2013;2013:753656.

8. Wang D, Couture R, Hong Y. Activated microglia in the spinal cord underlies diabetic neuropathic pain. Eur. J. Pharmacol. 2014;728:59-66.

9. Tsuda M. Microglia in the spinal cord and neuropathic pain. J. Diabetes Investig. 2016;7:17-26.

10. Ginhoux F, Greter M, Leboeuf M, Nandi S, See P, Gokhan S, Mehler MF, Conway SJ, Lai GN, Richard SE, et al. Fate mapping analysis reveals that adult microglia derive from primitive macrophages. Science. 2010;330:841-5.

11. Kawasaki Y, Zhang L, Cheng JK, Ji RR. Cytokine mechanisms of central sensitization: distinct and overlapping role of interleukin-1 $\beta$, interleukin-6, and tumor necrosis factor-a in regulating synaptic and neuronal activity in the superficial spinal cord. J Neurosci. 2008;28:5189-94.

12. Popiolek BK, Mika J. Targeting the microglial signaling pathways: new insights in the modulation of neuropathic pain. Curr Med Chem. 2016;23: 2908-28.

13. Tang T, Liu L, Xu D, Zhang W, Zhang Y, Zhou J, Huang W. Interaction between astrocytic colony stimulating factor and its receptor on microglia mediates central sensitization and behavioral hypersensitivity in chronic post ischemic pain model. Brain Behav. Immun. 2018;68:248-60.

14. Zhou D, Zhang S, Hu L, Gu YF, Cai Y, Wu D, Liu WT, Jiang CY, Kong X, Zhang GQ. Inhibition of apoptosis signal-regulating kinase by paeoniflorin attenuates neuroinflammation and ameliorates neuropathic pain. J. Neuroinflammation. 2019;16:83.

15. Shen Y, Ding Z, Ma S, Ding Z, Zhang Y, Zou Y, Xu F, Yang X, Schäfer MKE, Guo Q, et al. SETD7 mediates spinal microgliosis and neuropathic pain in rat model of peripheral nerve injury. Brain Behav. Immun. 2019;82:382-95.

16. Illisa AM, Gist AC, Zhang H, Kosturakis AK, Dougherty PM. Chemokine CCL2 and its receptor CCR2 in the dorsal root ganglion contribute to oxaliplatininduced mechanical hypersensitivity. Pain. 2018;159:1308-16.

17. Zhang ZJ, Jiang BC, Gao YJ. Chemokines in neuron-glial cell interaction and pathogenesis of neuropathic pain. Cell Mol Life Sci. 2017;74:3275-91.

18. Sommer C, Leinders M, Üçeyler N. Inflammation in the pathophysiology of neuropathic pain. Pain. 2018;159:595-602.

19. Hains BC, Waxman SG. Activated microglia contribute to the maintenance of chronic pain after spinal cord injury. J Neurosci. 2006:26:4308-17.

20. Ji RR, Suter MR. p38 MAPK, microglial signaling, and neuropathic pain. Mol Pain. 2007;3:33.

21. Zhuang ZY, Gerner P, Woolf CJ, Ji RR. ERK is sequentially activated in neurons, microglia, and astrocytes by spinal nerve ligation and contributes to mechanical allodynia in this neuropathic pain model. Pain. 2005;114:14959

22. Mollereau C, Mouledous L, Lapalu S, Cambois G, Moisand C, Butour JL, Meunier JC. Distinct mechanisms for activation of the opioid receptor-like 1 and kappa-opioid receptors by nociceptin and dynorphin A. Mol. Pharmacol. 1999:55:324-31.

23. Li TF, Fan H, Wang YX. Aconitum-derived bulleyaconitine A exhibits antihypersensitivity through direct stimulating dynorphin A expression in spinal microglia. J. Pain. 2016:17:530-48.

24. Wahlert A, Funkelstein L, Fitzsimmons B, Yaksh T, Hook V. Spinal astrocytes produce and secrete dynorphin neuropeptides. Neuropeptides. 2013;47: 109-15.

25. Frank DA, Greenberg ME. CREB: a mediator of long-term memory from mollusks to mammals. Cell. 1994;79:5-8.

26. Altarejos JY, Montminy M. CREB and the CRTC co-activators: sensors for hormonal and metabolic signals. Nat. Rev. Mol. Cell. Biol. 2011;12:141-51.

27. Lonze BE, Ginty DD. Function and regulation of CREB family transcription factors in the nervous system. Neuron. 2002;35:605-23.

28. McGrath JC, Lilley E. Implementing guidelines on reporting research using animals (ARRIVE etc.): new requirements for publication in BJP. Br. J. Pharmacol. 2015;172:3189-93.

29. Jiang L, Pan CL, Wang CY, Liu BQ, Han Y, Hu L, Liu L, Yang Y, Qu JW, Liu WT. Selective suppression of the JNK-MMP2/9 signal pathway by tetramethylpyrazine attenuates neuropathic pain in rats. J. Neuroinflammation. 2017;14:174.
30. Lee $\mathrm{CH}$, Yan BC, Yooet KY. Ischemia-induced changes in glucagon-like peptide-1 receptor and neuroprotective effect of its agonist, exendin-4, in experimental transient cerebral ischemia. J. Neurosci. Res. 2011;89:1103-13.

31. Hu M, Xu CB, Yang C, Zuo HL, Chen CJ, Zhang D, Shi GN, Wang WJ, Shi JG, Zhang TT. Discovery and evaluation of ZT55, a novel highly-selective tyrosine kinase inhibitor of JAK2V617F against myeloproliferative neoplasms. J Exp Clin Cancer Res. 2019;38:49

32. Lv GY, Dj S, Zhang JW, Xie XX, Wu XQ, Tian JW, Yan CH, Wang HB, Fu FH. LX2-32C, a novel semi-synthetic taxane, exerts antitumor activity against prostate cancer cells in vitro and in vivo. Acta. Pharm. Sin. B. 2017;7:52-8.

33. Nyirimigabo $E, X U Y, L i$ Y. A review on phytochemistry, pharmacology and toxicology studies of Aconitum. J. Pharm. Pharmacol. 2015;67:1-19.

34. Ji R, Gereau RW, Malcangio M, Strichartz GR. MAP kinase and pain. Brain Res. Rev. 2009;60:135-48

35. Moller T, Bard F, Bhattacharya A, Biber K, Campbell B, Dale E, Eder C, Gan L, Garden GA, Hughes ZA, et al. Critical data-based re-evaluation of minocycline as a putative specific microglia inhibitor. Glia. 2016;64:1788-94.

36. Huang Q, Mao XF, Wu HY, Liu H, Sun ML, Wang X, Wang YX. Cynandione A attenuates neuropathic pain through p38 $\beta$ MAPK-mediated spinal microglial expression of $\beta$-endorphin. Brain Behav. Immun. 2017;62:64-77.

37. Li TF, Wu HY, Wang YR, Li XY, Wang YX. Molecular signaling underlyingbulleyaconitine $\mathrm{A}$ (BAA)-induced microglial expression of prodynorphin. Sci. rep. 2017;7:45056.

38. Canon E, Cosgaya JM, Scsucova S, Aranda A. Rapid effects of retinoic acid on CREB and ERK phosphorylation in neuronal cells. Mol. Biol. Cell. 2004;15: 5583-92.

39. Stein C, Machelska H. Modulation of peripheral sensory neurons by the immune system: implications for pain therapy. Pharmacol Rev. 2011;63:86081.

40. Mélik PS, Rivat C, Rostène W, Réaux-Le GA. Opioid and chemokine receptor crosstalk: a promising target for pain therapy? Nat Rev Neurosci. 2015;16:6978.

41. Ji L, Chen Y, Wei H, Feng H, Chang R, Yu D, Wang X, Gong X, Zhang M. Activation of alpha7 acetylcholine receptors reduces neuropathic pain by decreasing dynorphin A release from microglia. Brain Res. 2019;15:57-65.

42. Brust A, Croker DE, Colless B, Ragnarsson L, Andersson A, Jain K, GarciaCaraballo S, Castro J, Brierley SM, Alewood PF, et al. Conopeptide-derived Kopioid agonists (Conorphins): potent, selective, and metabolic stable dynorphin A mimetics with antinociceptive properties. J Med Chem. 2016; 59:2381-95.

43. Wang C, Sun D, Liu C, Zhu C, Jing X, Chen S, Liu C, Zhi K, Xu T, Wang H, et al. Mother root of Aconitum carmichaelii Debeaux exerts antinociceptive effect in complete Freund's adjuvant-induced mice: roles of dynorpin/ kappa-opioid system and transient receptor potential vanilloid type-1 ion channel. J Transl Med. 2015;13:284.

44. Sun ML, Ao JP, Wang YR, Huang Q, Li TF, Li XY, Wang YX. Lappaconitine, a C18-diterpenoid alkaloid, exhibits antihypersensitivity in chronic pain through stimulation of spinal dynorphin A expression. Psychopharmacology. 2018;9:2559-71.

\section{Publisher's Note}

Springer Nature remains neutral with regard to jurisdictional claims in published maps and institutional affiliations.

Ready to submit your research? Choose BMC and benefit from

- fast, convenient online submission

- thorough peer review by experienced researchers in your field

- rapid publication on acceptance

- support for research data, including large and complex data types

- gold Open Access which fosters wider collaboration and increased citations

- maximum visibility for your research: over $100 \mathrm{M}$ website views per year

At BMC, research is always in progress.

Learn more biomedcentral.com/submissions 